09

\title{
Возбуждение поверхностных волн в плоскослоистых структурах и разработка модуляторов света
}

\author{
(С) А.Б. Петрин, ${ }^{1,2}$ О.Д. Вольпян, ${ }^{2}$ А.С. Сигов ${ }^{3}$ \\ ${ }^{1}$ Объединенный институт высоких температур РАН, \\ 125412 Москва, Россия \\ ${ }^{2}$ ООО «Научно-производственный комплекс Фотрон-Авто», \\ 117342 Москва, Россия \\ ${ }^{3}$ Московский технологический университет (МИРЭА), \\ 119454 Москва, Россия \\ e-mail: a_petrin@mail.ru
}

(Поступило в редакцию 23 июня 2017 г.)

На основе разработанного теоретического метода исследования отражения плоской электромагнитной волны от плоскослоистой структуры с анизотропными пленками предложены конструкции модуляторов света и перестраиваемых датчиков. Низкие напряжения переключения модуляторов в предложенных устройствах позволяют встраивать их в современные электронные интегральные схемы, создавать высокоскоростные оптические каналы передачи данных как между составными частями отдельной интегральной схемы, так и между удаленными устройствами.

DOI: $10.21883 /$ JTF.2018.03.45603.2400

\section{Введение}

В настоящее время большое внимание уделяется перспективным устройствам, использующим поверхностные плазмонные волны [1-4]. Такие волны распространяются вдоль поверхности металла, локализуясь вблизи его поверхности [5,6]. Распространенным способом возбуждения поверхностных плазмонов является метод Кречмана [7].

В рамках схемы Кречмана [8] (рис. 1) согласование поверхностной плазмонной волны 1 на поверхности металлической пленки 2 и падающей волны 3 в свободном пространстве осуществляется с помощью стеклянной призмы 4.

Линейно поляризованная волна $\mathbf{E}^{i}$ от лазера падает на металлическую пленку со стороны призмы. Вектор электрического поля падающей волны направлен в плоскости падения ( $p$-поляризация). Призма необходима для согласования падающей свободной волны и поверхностной плазмонной волны по волновому числу. Волновое число поверхностной плазмонной волны больше волнового числа плоской волны в свободном пространстве той же частоты $[9,10]$. Чтобы обеспечить равенство волновых чисел падающей 3 , отраженной 5 и поверхностной плазмонной 1 волн вдоль поверхности металла (рис. 1), необходимо, чтобы падающая волна подходила к пленке из оптически более плотной среды, где волновое число больше, чем в свободном пространстве. Показатель преломления призмы и угол падения подбираются таким образом, чтобы в отсутствии возбуждения поверхностных плазмонов по закону Снелиуса наблюдалось бы полное внутреннее отражение.

Эксперименты показывают, что если угол падения соответствует согласованию по волновому числу пада- ющей и поверхностной плазмонной волн, наблюдается резкое падение коэффициента отражения. Например, при длине падающей волны в вакууме $\lambda=633 \mathrm{~nm}$ и толщине пленки серебра, приблизительно равной $53.8 \mathrm{~nm}$ (эта величина зависит от конкретного значения показателя преломления призмы и частоты падающей волны), наблюдается сильное изменение коэффициента отражения практически от единицы до нуля в окрестности угла падения, соответствующего согласованию свободной и поверхностной плазмонной волн. При небольшом отклонении угла падения от угла согласования коэффициент отражения возвращается практически от нуля к единичному значению.

Так как поле поверхностной плазмонной волны сосредоточено в тонком слое вблизи поверхности металла, характер отражения в схеме Кречмана сильно зависит от изменений показателя преломления в тонком слое

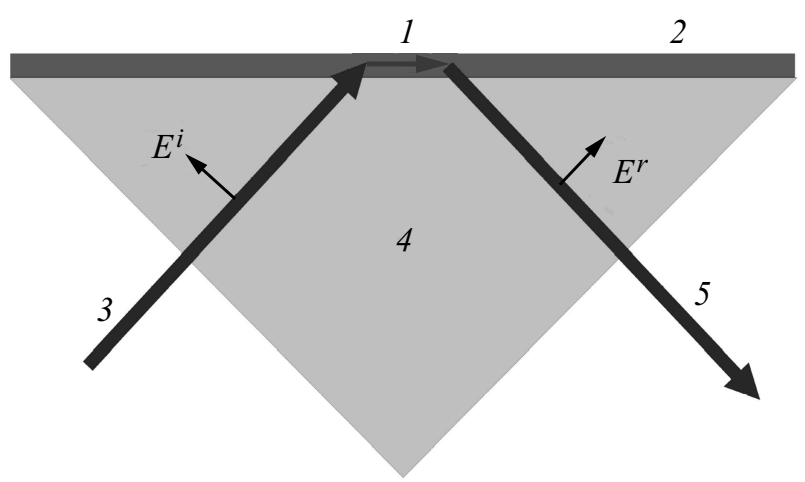

Рис. 1. Возбуждение поверхностных плазмонов 1 на поверхности металлической пленки 2 по схеме Кречмана. Падающая волна 3 в призме 4 порождает поверхностную плазмонную волну 1 и отраженную волну 5 . 
вблизи поверхности. На этом основано применение данной схемы для создания различного рода датчиков, обладающих высокой чувствительностью к изменению состояния вещества на поверхности [11]. Исследования показали, что чувствительность определяется угловой шириной минимума отражения [12], которая, в свою очередь, определяется поглощением в металле пленки. Чем меньше поглощение в металле, тем у́же минимум.

Чтобы увеличить чувствительность датчиков за счет уменьшения угловой ширины минимума, было предложено уменьшать толщину пленки металла. Оказалось, что у поверхностной плазмонной волны в тонкой пленке затухание уменьшается с уменьшением толщины пленки. Для согласования поверхностной волны в схеме Кречмана потребовалось введение дополнительного согласующего слоя между призмой и металлической пленкой [13-16], причем в некоторых случаях даже многопленочного слоя $[17,18]$.

Однако известно, что поверхностные волны могут распространяться и в диэлектрических пленках планарных волноводов [19]. В связи с этим можно предположить, что в схеме Кречмана можно возбуждать поверхностную волну (не обязательно плазмонную), которая согласуется по волновому числу с падающей волной. На этой идее строится подход настоящей работы по построению нового вида электрически подстраиваемых сенсоров и модуляторов света.

Подход состоит в том, что в качестве волновода поверхностной волны можно использовать пленку электрооптического вещества, например ниобата лития, расположенную между двумя слоями очень тонких оптически прозрачных или металлических электропроводящих пленок-электродов. Проводящие пленки играют роль пластин конденсатора, в котором находится электрооптическая пленка. Подача напряжения на проводящие пленки будет создавать электрическое поле, изменяющее тензор диэлектрической проницаемости электрооптической пленки и характер отражения в многопленочной системе. Как будет показано ниже, устройства с такой многопленочной структурой могут обеспечивать переключение с режима практически полного поглощения на режим полного отражения при подаче на пленкиэлектроды низкого напряжения, порядка одного вольта. Это позволит совместить такие устройства с интегральными электронными цифровыми микросхемами.

Следует отметить, что, в случае устройств конечных размеров (в плоскости многопленочной структуры) пучок падающей волны ограничен по сечению (апертуре), поэтому отражение будет происходить не совсем так, как в случае бесконечной структуры и идеально плоских волн. Однако, как показали численные расчеты отражения ограниченных по апертуре пучков [20,21], основные закономерности отражения сохраняются. В настоящей работе отражение ограниченных пучков рассматриваться не будет.

\section{Задача падения плоской электромагнитной волны на изотропно-анизотропную плоскослоистую структуру}

Задача падения плоской электромагнитной волны с поляризацией в плоскости падения на плоскослоистую структуру хорошо известна, поэтому мы не будем подробно останавливаться на изложении метода. В настоящей работе мы использовали метод, аналогичный методу работы [22]. В результате вычислений мы находили коэффициент отражения по полю $R$, а также модуль коэффициента усиления поля $A$ на внешней (свободной) границе многослойной конструкции по отношению к полю падающей на многопленочную структуру волны. Отметим особо, что в настоящей работе мы исследовали возбуждение поверхностной волны в слоистой структуре в кречмановской схеме возбуждения поверхностных волн, при которой волна не проходит через структуру (угол падения больше угла полного внутреннего отражения по закону Снелиуса).

\section{Нахождение оптимальных толщин пленок для случая применения пленки ниобата лития в качестве электрооптической среды}

В качестве электрооптической пленки была взята пленка ниобата лития, причем направления осей тензора диэлектрической проницаемости в пленке выбирались таким образом, чтобы ось необыкновенной волны была направлена перпендикулярно пленке (рис. 2). Длина падающей волны в вакууме принималась равной $\lambda=633 \mathrm{~nm}$.

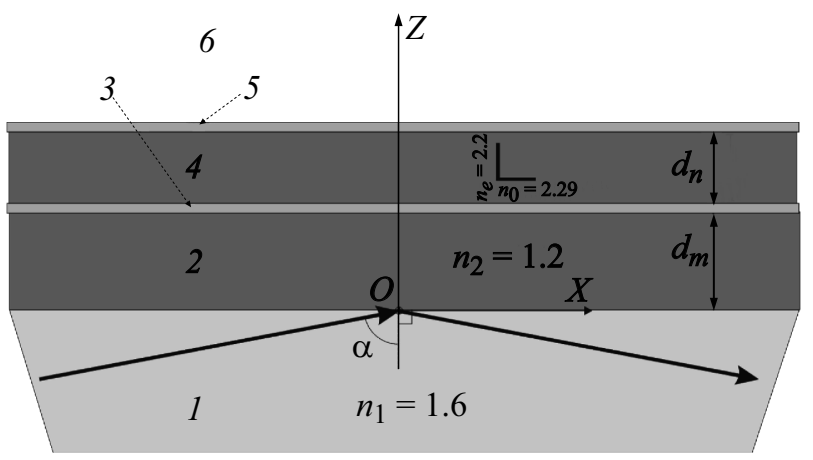

Pис. 2. Геометрия многопленочной структуры. Плоская волна $p$-поляризации падает из призмы 1 на четырехслойную структуру, состоящую из согласующей пленки 2, первого электрода 3 , пленки ниобата лития 4 и пленки второго электрода 5. За пленкой находится вакуум 6 . Показаны ориентации осей тензора диэлектрической проницаемости пленки ниобата лития и толщины пленок $d_{n}$ и $d_{m}$, по которым проводится оптимизация. 
Тензор диэлектрической проницаемости в пленке ниобата лития в системе координат рис. 2 записывался формулой

$$
\varepsilon_{4}=\left[\begin{array}{ccc}
n_{0}^{2}\left(1-\alpha n_{0}^{2} E\right) & 0 & 0 \\
0 & n_{0}^{2}\left(1-\alpha n_{0}^{2} E\right) & 0 \\
0 & 0 & n_{e}^{2}\left(1-\gamma n_{e}^{2} E\right)
\end{array}\right],
$$

где $E$ - напряженность управляющего электрического поля между пленками-электродами 3 и 5 (рис. 2), а для ниобата лития принимались следующие величины коэффициентов: $\alpha=r_{13}=r_{23}=8.6 \mathrm{pm} / \mathrm{V}$, $\gamma=r_{33}=30.8 \mathrm{pm} / \mathrm{V}, n_{0}=2.29, n_{e}=2.20$.

Необходимо отметить, что не при всех толщинах пленок, показанных на рис. 2, возможен режим поверхностной волны. Для его нахождения использовался следующий вычислительный метод оптимизации структуры.

- В рассматриваемой структуре фиксировались следующие параметры: показатель преломления призмы $n_{1}=1.6$; показатель преломления согласующего слоя $n_{2}=1.2$; параметры тензора диэлектрической проницаемости ниобата лития; комплексная диэлектрическая проницаемость проводящих электродов принималась равной $\varepsilon_{3}=\varepsilon_{5}=1.8^{2}+i 0.002$, а их толщина фиксировалась равной $10 \mathrm{~nm}$.

- С помощью двух параметров: толщины пленки ниобата лития $d_{n}$ и толщины согласующего слоя $d_{m}$ (рис. 2) искался оптимум структуры: фиксировалось два значения $\left(d_{n}, d_{m}\right)$, вычислялась зависимость $A=A(\alpha)$ в диапазоне углов полного отражения, находился максимум. Затем значение максимума вычислялось для другой пары значений $\left(d_{n}, d_{m}\right)$ и т. д. Максимум максиморум для всех пар $\left(d_{n}, d_{m}\right)$, взятых в широком диапазоне значений толщин, давал нам искомый оптимальный режим поверхностной волны с максимальной концентрацией поля на внешней поверхности многослойной структуры.

Результат описанной оптимизации представлен в табл. 1.
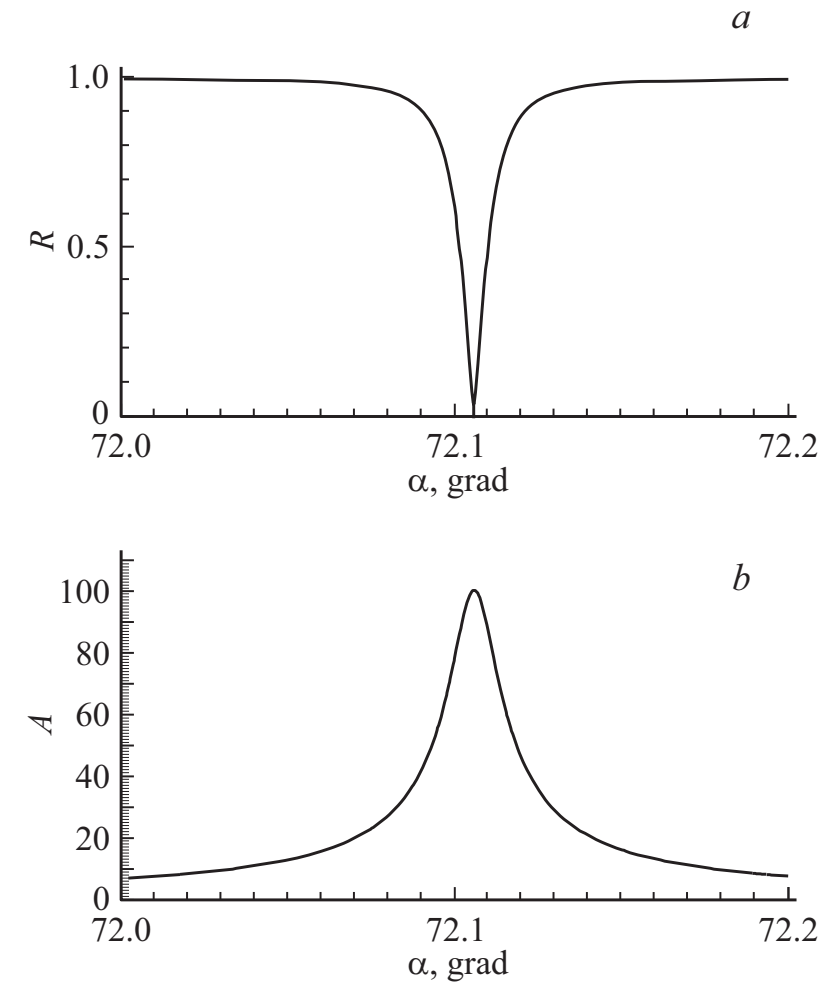

Рис. 3. Зависимость коэффициента отражения $R$ по полю $(a)$ и коэффициента усиления $A$ поля $(b)$ от угла падения плоской волны на многопленочную структуру (табл. 1).

На рис. 3 показана зависимость $R=R(\alpha)$ и $A=A(\alpha)$ для оптимальной структуры табл. 1 . Из рисунков видно, что резкое изменение коэффициента отражения происходит при малом отклонении угла от угла минимума (угловая ширина изменения $R$ и $A$ мала). Это позволяет сделать заключение, что датчики, построенные на подобной структуре, будут иметь высокую чувствительность. Для сравнения на рис. 4 показаны зависимости $R=R(\alpha)$

Таблица 1.

\begin{tabular}{|c|c|c|c|}
\hline $\begin{array}{c}\text { Порядковый } \\
\text { номер среды, } j\end{array}$ & Название слоя & $\begin{array}{c}\text { Относительная диэлектрическая } \\
\text { проницаемость }\end{array}$ & $\begin{array}{c}\text { Толщина пленки, } \\
\text { nm }\end{array}$ \\
\hline 1 & Призма & $1.6^{2}$ & $\begin{array}{c}\text { Полубесконечная } \\
\text { среда }\end{array}$ \\
\hline 2 & Согласующий слой & $1.2^{2}$ & 427 \\
\hline 3 & Прозрачный электрод & $1.8^{2}+i 0.002$ & 10 \\
\hline 4 & Ниобат лития & $\begin{array}{c}\varepsilon_{14}=n_{0}^{2}=2.2^{2}, \varepsilon_{4}=n_{e}^{2}=2.29^{2} \\
(\text { без поля между электродами })\end{array}$ & 327 \\
\hline 5 & Прозрачный электрод & $1.8^{2}+i 0.002$ & 10 \\
\hline 6 & $\begin{array}{c}\text { Свободное пространство } \\
(\text { воздух })\end{array}$ & 1 & $\begin{array}{c}\text { Полубесконечная } \\
\text { среда }\end{array}$ \\
\hline
\end{tabular}


Таблица 2.

\begin{tabular}{|c|c|c|c|}
\hline $\begin{array}{c}\text { Порядковый } \\
\text { номер среды, } j\end{array}$ & Название слоя & $\begin{array}{c}\text { Относительная диэлектрическая } \\
\text { проницаемость }\end{array}$ & $\begin{array}{c}\text { Толщина пленки, } \\
\text { nm }\end{array}$ \\
\hline 1 & Призма & $1.6^{2}$ & $\begin{array}{c}\text { Полубесконечная } \\
\text { среда }\end{array}$ \\
\hline 2 & Серебряная пленка & $\varepsilon_{m}=-18.2+i 0.5$ & 53.8 \\
\hline 3 & $\begin{array}{c}\text { Свободное пространство } \\
\text { (воздух })\end{array}$ & 1 & $\begin{array}{c}\text { Полубесконечная } \\
\text { среда }\end{array}$ \\
\hline
\end{tabular}

и $A=A(\alpha)$ для серебряной пленки толщиной $53.8 \mathrm{~nm}$, расположенной на поверхности призмы (рис. 1). В такой структуре поверхностной волной является поверхностная плазмонная волна на поверхности металлической пленки $([10,22])$. Параметры такой однопленочной оптимизированной структуры приведены в табл. 2.

Сравнивая зависимости рис. 4 и зависимости для многопленочной структуры (рис. 3), можно заметить, что значения $A$ в максимуме для многопленочной структуры достигают значений $A_{\max } \approx 100.6$, что намного больше, чем в случае использования серебряной пленки при возбуждении поверхностных плазмонов в схеме Кречмана $\left(A_{\max } \approx 5.8\right)$ [22]. Это говорит о том, что в предложенной многопленочной структуре будет намного проще наблюдать нелинейные взаимодействия по сравнению с обычной схемой возбуждения поверхностной плазмонной волны [22].
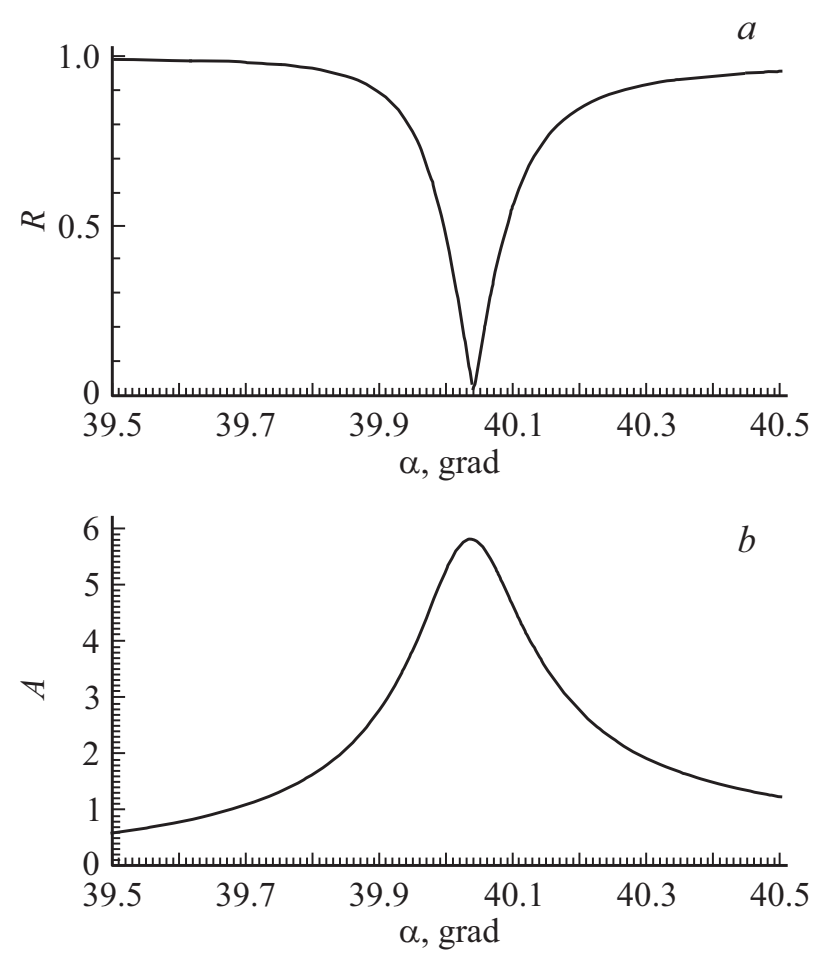

Pис. 4. Зависимость коэффициента отражения $R$ по полю $(a)$ и коэффициента усиления $A$ поля $(b)$ от угла падения плоской волны на серебряную пленку (табл. 2).

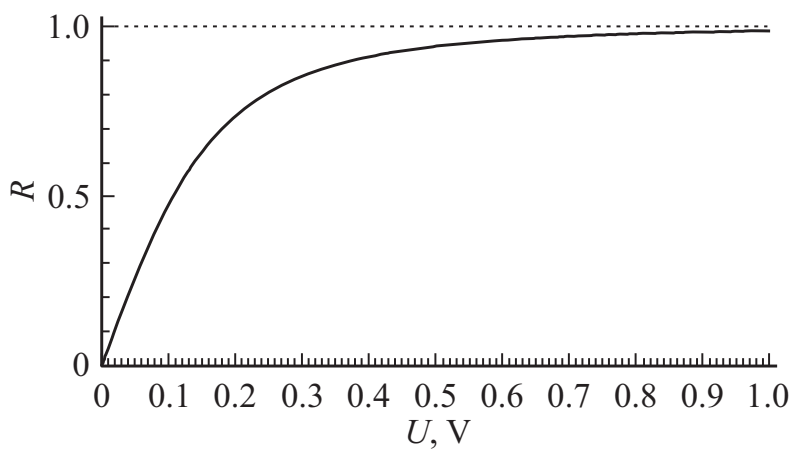

Рис. 5. Зависимость коэффициента отражения по полю от напряжения между пленками-электродами. В отсутствии напряжения система настроена на минимум отражения.

Имея в виду применение многопленочной структуры табл. 1 для создания модуляторов света, была найдена зависимость $R=R(U)$ (рис. 5), где $U-$ напряжение между пленками-электродами. При вычислении $R=R(U)$ угол падения волны $\alpha_{*}$ фиксировался на значении минимума зависимости $R=R(\alpha)$ при нулевом напряжении $U=0$. При изменении напряжения $U$ и соответственно напряженности поля $E$ между электродами изменялись тензор диэлектрической проницаемости электрооптической пленки и коэффициент отражения $R\left(\alpha_{*}\right)$ всей многопленочной структуры. Из рис. 5 видно, что для переключения из режима практически полного поглощения падающей волны на режим практически полного отражения необходимо подать на электроды напряжение всего порядка 1 V. Это дает возможность встраивать подобное устройство в стандартные цифровые электронные интегральные схемы.

\section{Заключение}

В работе предложен метод построения многопленочных структур с поверхностными волнами, которые обладают в том числе возможностью изменения параметров распространения поверхностной волны с помощью электрооптических элементов. Полученные устройства могут быть применены для создания перестраиваемых электрическими сигналами датчиков, электрооптических 
модуляторов и других устройств, совместимых с цифровыми и аналоговыми интегральными микросхемами.

Работа выполнена при финансовой поддержке Министерства образования и науки Российской Федерации (научный проект „Оптические транзисторы на основе метаматериалов“". Соглашение о предоставлении субсидии с Министерством образования и науки Российской Федерации от 29.09.2016 г., № 14.577.21.0219, уникальный идентификатор ПНИЭР RFMEFI 57716 X 0219).

\section{Список литературы}

[1] Homola J., Yee S.S., Gauglitz G. // Sensors and Actuators B. 1999. Vol. 54. P. 3.

[2] Barnes W.L., Dereux A., Ebbesen T.W. // Nature. 2003. Vol. 424. P. 824.

[3] Homola J. // Chem. Rev. 2008. Vol. 108. P. 462.

[4] Spoto G., Minunni M. // J. Phys. Chem. Lett. 2012. Vol. 3. P. 2682.

[5] Raether H. Surface Plasmons. Berlin: Springer-Verlag, 1988. $353 \mathrm{p}$.

[6] Barnes W.L. // J. Opt. A: Pure Appl. Opt. 2006. Vol. 8. P. S87.

[7] Kretschmann E., Raether H.Z. // Naturforsch. 1968. A. Vol. 23. P. 2135.

[8] Piliarik M., Homola J. // Opt. Express. 2009. Vol. 17. N 19. P. 16505.

[9] Liedberg B., Nylander C., Lundstrom I. // Sensors and Actuators. 1983. Vol. 4. P. 299.

[10] Liedberg B., Nylander C., Lundstrom I. // Biosensors \& Bioelectronics. 1995. Vol. 10.

[11] Garabedian R., Gonzalez C., Richards J. et al. // Sensors and Actuators A. 1994. Vol. 43. P. 202.

[12] Yeatman E.M. // Biosensors \& Bioelectronics. 1996. Vol. 11. P. 635.

[13] Sarid D. // Phys. Rev. Lett. 1981. Vol. 47. P. 1927.

[14] Matsubara K., Kawata S., Minami S. // Opt. Lett. 1990. Vol. 15. P. 75.

[15] Yang F., Bradberry G.W., Sambles J.R. // Phys. Rev. Lett. 1991. Vol. 66. P. 2030

[16] Kessler M.A., Hall E.A.H. // Thin Solid Films. 1996. Vol. 272. P. 161.

[17] Nenninger G.G., Tobiska P., Homola J., Yee S.S. // Sens. Actuators B. 2001. Vol. 74. P. 145.

[18] Toyama S., Doumae N., Shoji A., Ikariyama Y. // Sens. Actuators B. 2000. Vol. 65. P. 32.

[19] Airoudj A., Debarnot D., Beche B., Poncin-Epaillard F. // Anal. Chem. 2008. Vol. 80. P. 9188.

[20] Irvine S.E., Dechant A., Elezzabi A.Y. // Phys. Rev. Lett. 2004. Vol. 93. P. 184801.

[21] Irvine S.E., Elezzabi A.Y. // Phys. Rev. A. 2006. Vol. 73. P. 013815

[22] Новотный Л., Хехт Б. Основы нанооптики. Пер. с англ. / Под ред. В.В. Самарцева. М.: Физматлит, 2009. 484 с. 